\title{
A Novel Energy Efficient Routing Protocols for Wireless Sensor Networks Using Spatial Correlation Based Collaborative Medium Access Control Combined with Hybrid MAC
}

\author{
A. Rajeswari \\ Department of ECE, \\ Coimbatore Institute of Technology, Coimbatore (India) \\ Tel: +91-9865907804 E-mail: rajeswari@ cit.edu.in \\ P. T. Kalaivaani \\ Research Scholar, \\ Anna University of Technology, Coimbatore (India) \\ Tel: +91-9962099630 E-mail: ptkalaivaani@gmail.com
}

Received: December 1, 2011 Accepted: December 15, 2011 Published: December 31, 2011 DOI: 10.5296/npa.v3i4.1296 URL: http://dx.doi.org/10.5296/npa.v3i4.1296

\begin{abstract}
Wireless Sensor Networks (WSNs) are wireless networks consisting of a group of small, inexpensive nodes, which collect and disseminate significant data. Nodes in a wireless Sensor Networks have various energy and computational constraints due to their inexpensive, random method of deployment. Wireless Sensor Networks are utilized in a wide range of applications including battlefield surveillance, smart home environments, habitat exploration of animals and vehicle tracking. Energy Efficiency is one critical issue for wireless sensor networks because many sensors are equipped with batteries that have limited life time. This paper introduces the concept of combining two Medium Access Control (MAC) Protocols such as Spatial Correlation based Collaborative Medium Access Control (CCMAC) and Hybrid Medium Access Control (HMAC) which will increase the energy efficiency of the Network. The four various routing protocols explored in this work are AOMDV,AODV, DSR,DSDV. In this paper, comparison of energy efficient routing protocols for Wireless Sensor Networks using Spatial Correlation Based Collaborative Medium Access Control
\end{abstract}


(CCMAC) combined with Hybrid Medium Access Control (HMAC) is proposed. Simulation results have been obtained by using Ns2. From the results, the performance of AOMDV is better compared to the other three protocols such as AODV,DSR and DSDV.

Keywords: Adhoc On Demand Distance Vector Routing (AODV), Adhoc On Demand Multipath Distance Vector Routing (AOMDV), Dynamic Source Routing (DSR), Destination Sequenced Distance Vector (DSDV), Hybrid Medium Access Control (HMAC), Spatial Correlation based Collaborative Medium Access Control (CCMAC), Medium Access Control (MAC), Wireless Sensor Network (WSN).

\section{Introduction}

An emblematic sensor node in WSN consists of a wireless communication unit and a processing unit, a sensing unit, and a power supply unit. The energy source of a node is generally considered non-rechargeable. Therefore energy conservation is a major research challenge in WSN. For practical deployment, Wireless Sensor Networks must be energy efficient. Using Energy as a vital resource in WSNs, Several MAC protocols have already been proposed to get higher energy efficiency during long idle period of the sensors [3]. Each sensor node in a WSN has three basic units; The sensing unit can sense various phenomena including light, temperature, sound and motion around its location. Process unit packetizes the sensed data and the transmission unit sends the packetized data to a base station, data receiving and data sending process are carried out by communication unit. Sensing unit consumes less energy than communication unit because most of the energy is consumed during data receiving and data sending which are carried out in communication unit. In Wireless Sensor Networks, an energy efficient medium access control protocol is required for obtaining higher energy efficiency in very difficult operating conditions, where node and link failures are common. The MAC protocol manages radio transmissions and receptions on a shared wireless medium. Therefore MAC has a very high effect on network performance and energy consumption. In this paper, a Novel Energy Efficient routing protocols for Wireless Sensor Networks using Spatial Correlation based Collaborative Medium Access Control combined with Hybrid MAC has been proposed. In the Proposed wok spatial correlation is exploited on Medium Access Control. Spatial Correlation based Collaborative Medium Access Control regulates the redundant transmission from closely located sensor nodes. Another MAC protocol is Hybrid MAC (HMAC), which is suitable for wireless sensor networks interms of energy efficiency, Latency. HMAC is simple and Scalable and doesn't have to maintain the neighbor node's information also it needs low cost to design. The Related work is clearly explained in Section 2. Section 3 deals with problem description where the spatial correlation in WSN, Distortion constraint and simulation results for distortion constraint and spatial correlation based CCMAC and Hybrid MAC, Routing Protocols, System Model concepts are discussed in detail. In Section 4, Spatial Correlation based Iterative Node Selection Algorithm (SCINS) and Pseudo Code for SCINS are explained. In Section 5, Results has been discussed. It includes Comparison of End to End delay, Packet Delivery Ratio and Throughput and Energy 
Consumption with AOMDV,AODV, DSR, DSDV. Conclusion and future work has been discussed in Section 6.

\section{Related Work}

A brief literature survey is presented in the following section:

Due to the spatial correlation between the sensor nodes, it may not be necessary for every sensor nodes to transmit its data to sink nodes [1]. A drawback of the work is that only one type of phenomenon is sensed by the sensor nodes in a network [1]. A Spatial Correlation Based Medium Access Control protocol is developed to filter out redundant data. Therefore energy consumption of the network is reduced [2]. The specific requirement of wireless sensor real time MAC protocol is developed and the taxonomy for real-time MAC protocol in wireless sensor networks is proposed [3]. The concepts of MAC layer related sensor network properties and the reasons of energy waste are discussed [4]. An energy aware management of sensor networks that maximizes the lifetime of the sensors and reduces energy consumption by dynamically assigning the routes and arbitrates medium access is discussed [5]. Under minimum energy usage the spatio-temporal sampling rate of the network is determined [6]. A novel channel reservation technique is proposed to reduce end to end delay. HMAC protocol with an embedded cross layer optimization technique is also proposed and QOS based Routing is investigated in this paper [12]. The performance of AODV and AOMDV protocols are evaluated [13].

\section{Problem Description}

A wireless radio is the most energy consuming unit of a sensor node in WSN. It can operate in four different states. They are transmit, receive and idle and sleep. When the nodes are in active state, almost all the nodes consume the same energy. There are several ways to reduce the energy consumption in WSN. One way of reducing the energy consumption is, by using only a required set of nodes as active and thereby reducing redundant network traffic, decreasing packet forwarding delay, to help in avoiding packet collisions. The other way is to put few sensor nodes into sleep state and use only necessary node to be in active mode for sensing and communication.

In this paper, a Novel Energy Efficient routing protocols for Wireless Sensor Networks using Spatial Correlation based Collaborative Medium Access Control combined with Hybrid MAC has been proposed. In the proposed work, the routing protocols such as AOMDV, AODV, DSR and DSDV are considered only to analyze the behavior of CC-MAC protocol combined with HMAC protocol. Also the proposed work considers spatial correlation which is exploited on the medium access control layer [1]. Network Simulator (Ns2) has been used for simulation of comparison of energy efficient routing protocols for wireless sensor network using Spatial Correlation based Collaborative Medium Access Control combined with HMAC. 


\subsection{Spatial Correlation in Wireless Sensor Networks}

Spatial Correlation between the sensor nodes helps to prevent redundant data during transmission. In wireless sensor network, when an event occurs in a sensor field, the nodes which are very nearer to that event area detect the event information and it is sensed by the neighborhood nodes. Every node transmits its own data to sink which is highly correlated that results in redundant transmission. It is not requisite for all the nodes in the sensor field to send the data to the sink. Redundant transmission in a network is reduced by decreasing the selecting the subset of sensor nodes. The subset nodes efficiently transmit the data to sink. The filtration of redundant data and the maximization of network lifetime are increased by designing the efficient MAC protocol. The spatial correlation region is defined as the region in which all the sensor nodes send the readings which are similar in nature and therefore it is enough to send a single report to represent the correlation region [8].

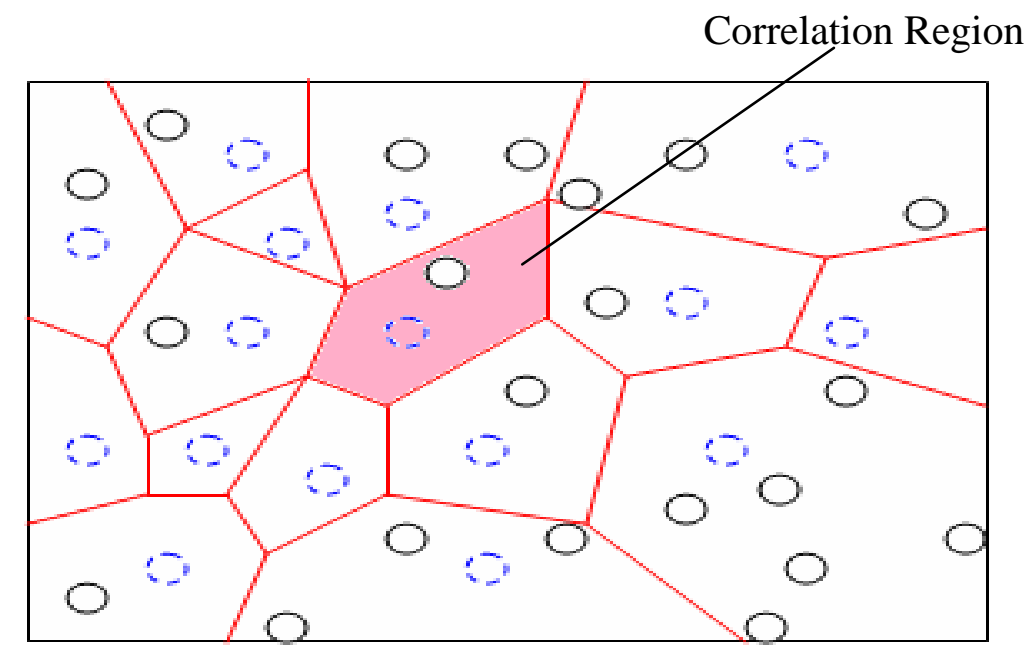

Figure 1. Representation of Correlation Region

Figure 1. shows the representation of correlation region.

$$
\operatorname{corr}\left\{S_{i,}, S_{j}\right\}=\rho_{i, j}=K_{v}\left(d_{i, j}\right)=\frac{E\left[S_{i}, S_{j}\right]}{\sigma_{s}^{2}}
$$

Equation (1) gives the correlation between the two nodes $n_{i}$ and $n_{j}$ located at the coordinates $S_{i}, S_{j}$. where $d_{i, j}=\rho_{s i}-\rho_{s j}$. where $\rho$ denotes the distance between nodes $n_{i}$ and $n_{j}$ respectively, and $k_{v}(\cdot)$ is the correlation model or covariance model. The covariance function is assumed to be non-negative and decreases monotonically with the distance. Covariance model is categorized into four types, namely, i) Spherical Model, ii) Power Exponential Model, iii) Rational Quadratic Model, iv) Matern Model 
In this paper Power exponential model has been considered [1]. The covariance function is given by

$$
K_{v}^{P E}(d)=e^{\left(-d / \theta_{1}\right)^{\theta_{2}}} ; \theta_{1}>0, \theta_{2} \in(0,2]
$$

In Equation (2), $\mathrm{K}_{\mathrm{v}}$ represents covariance function and PE represents power exponential model. For $\theta_{2}=1$, the model becomes exponential, while for $\theta_{2}=2$ the model becomes squared exponential. The parameter $\theta_{1}$ is important because it controls the relationship between the distance $d_{i, j}$ and $\rho_{i, j}$.

\subsection{Distortion Constraint and Simulation results for distortion constraint}

Distortion is one of the reliability constraint. The distortion increases when the sensor nodes fail to report the event from within the defined correlation region. The correlation region is changed dynamically according to the observed reliability. At sink node, the distortion is given by,

$$
\mathrm{D}=\mathrm{E}[\mathrm{d}(\mathrm{S}, \hat{S})]
$$

where $\mathrm{D}$ is the Distortion value and $\mathrm{S}$ is the event and $\hat{S}$ is the estimated value of $\mathrm{S}$. Mean-squared error is used as the distortion metric

$$
D(M)=E\left[(S-\hat{S}(M))^{2}\right]
$$

$\mathrm{D}(\mathrm{M})$ shows the distortion achieved at the sink as a function of number of nodes $\mathrm{M}$ that send information to the sink and correlation coefficients $\rho_{(\mathrm{i}, \mathrm{j}) \text {. }}$ and $\rho_{(\mathrm{s}, \mathrm{i})}$ between nodes $\mathrm{n}_{\mathrm{i}}$ and $\mathrm{n}_{\mathrm{j}}$, and the event source $S$ and node $n_{i}$, respectively [1]. It is denoted as,

$$
\begin{gathered}
D(M)=\sigma_{s}^{2}-\frac{\sigma_{s}^{4}}{M\left(\sigma_{s}^{2}+\sigma_{N}^{2}\right)}\left(2 \sum_{i=1}^{M} \rho_{(s, i)}-1\right)+ \\
\frac{\sigma_{s}^{6}}{M^{2}\left(\sigma_{s}^{2}+\sigma_{N}^{2}\right)^{2}} \sum_{i=1}^{M} \sum_{j \neq i}^{M} \rho_{(i, j)}
\end{gathered}
$$

The wireless sensor network is implemented in Ns2 with fifty nodes in random deployment. Representative nodes are selected randomly among 100 nodes and the distortion function is calculated according to the locations of these nodes. From the simulation, the 
correlation coefficient between the sensor nodes and the event and also the average distortion are calculated, the distribution of the distortion for each number of representative nodes is shown in Figure 4. For the analysis, $\theta_{2}=1$ and $\theta_{1}=\{10,50,100,500,1000,5000,10000\}$ have been considered in the covariance model for the covariance function.

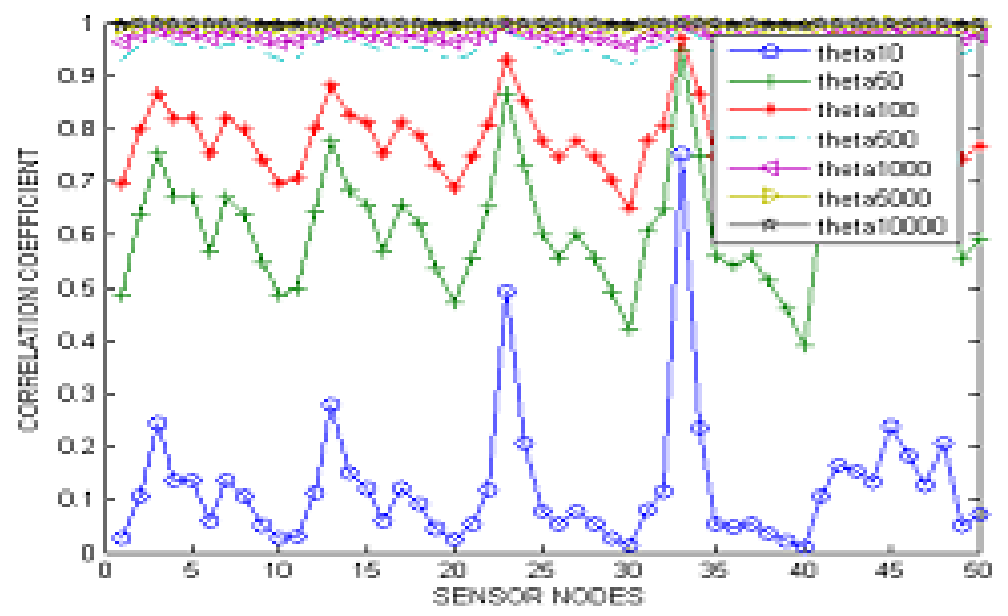

Figure 2. Correlation Coefficient between the sensor nodes and the event

Figure 2 shows the correlation coefficient between the sensor nodes and the event.

As shown in Figure 2 , the achieved distortion stays relatively constant when the number of representative nodes is decreased above 50. This behavior is due to the highly redundant data sent by the sensor nodes that are close to each other. In addition, with increasing $\theta_{1}$, the observed event distortion decreases since close nodes become less correlated with increasing $\theta_{1}$.

\subsection{Spatial Correlation based Collaborative MAC (CC-MAC) in WSN}

Spatial correlation based CC-MAC reduces the unnecessary channel access contention by filtering correlated data transmission. CC-MAC contains two primary components. They are Event MAC and Network MAC protocol. Event MAC filers out the correlation in sensor network and Network MAC protocol prioritize the route through packets. E MAC forms the correlation based clusters. N MAC prioritizes the route through packets when the medium access is in usage. The Data Packet Structure of CC - MAC protocol is given in Figure 3.

\begin{tabular}{|l|l|l|l|}
\hline MAC Header & FH & Frame Body & FCS \\
\hline Bits 240 & 1 & $0-18496$ & 32
\end{tabular}

Figure 3. Data Packet Structure

CC-MAC distributed operation is based only on $r_{\text {corr }}$, where $r_{\text {corr }}$ is the correlation 
radius. Sink sends the $r_{\text {corr }}$ value to each sensor node in the network. CC - MAC protocol has the capability to decrease redundant data and energy consumption. The representative node selection algorithm in CC-MAC protocol is random in nature. Total number of representative nodes is decreased automatically when the correlation value is increased.

\subsection{Hybrid MAC Protocol (HMAC)}

To overcome the short comings of Carrier Sense Multiple Access (CSMA) and Time division Multiple Access (TDMA), HMAC improves the channel utilization and latency under different network condition. HMAC introduces the concept of slotted frame structure in which slot for individual frame structures are dynamically shared. A slotted frame structure helps to improve energy efficiency. In HMAC, slotted frame structure needs time synchronization [12] with in each nodes two hop neighbour. HMAC also supports one hop broadcast mechanism. HMAC Frame Structure is given in Figure 4.

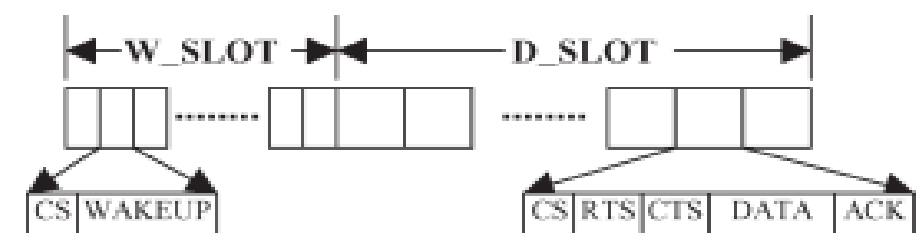

Figure 4. HMAC Frame Structure

\subsection{Routing Protocols}

In the proposed work, four different type of routing protocols are considered for the analysis purpose. They are Adhoc On demand distance vector routing protocol (AODV) and Adhoc on demand multipath distance vector routing protocol (AOMDV) and Dynamic Source Routing(DSR) and destination sequenced distance vector routing protocol (DSDV).Routing Protocols are classified into four different types they are proactive and reactive routing protocols.

Proactive Routing Protocols maintain fresh list of destinations and their routes by periodically distributing routing tables throughout the network. The main disadvantages are respective amount of data for maintenance and slow reaction on restructuring and failures. Reactive Protocols are also known as On-demand protocols. These type of protocols find a route on demand by flooding the network with route request packets. AODV is also appropriate for working in restrictive environments. It has the ability to intercommunicate with the endpoints which cannot be accessed directly. DSDV is an elementary and moderately less complex protocol which is a suitable fit for less dense network i.e. targeted to function exquisitely on small node density. DSDV does work, essentially, by sharing routing information with neighboring nodes, which is stacked away by each node in the form of tables. DSR uses source routing. The main feature of DSR is source routing. i.e, the sender knows the complete hop-by-hop route to the destination. These routes are stored in a route cache. The data packets carry the source route in the packet header. AOMDV is an extension of AODV 


\section{Al Macrothink}

protocol for computing multiple loop free and link disjoint paths. The routing entries for each destination contains list of next hops along with the corresponding hop count. The advantage of using AOMDV is that it allows intermediate nodes to reply for RREQ, which still selecting disjoint paths.

\subsection{System Model}

A model for wireless sensor network has been designed and shown in Figure 5. Event source is represented as $\mathrm{S}$. Total number of nodes available in an event area is N, Each node in the sensor field observes the noisy version of event information $X_{i}(n), S_{i}(n)$ is spatially correlated to event source $S$. Each node has to encode its observation for the purpose of reporting an event information to sink node. Sink is available at the other end which is responsible for decoding the information to get an estimated value of $\hat{S}$ [1].

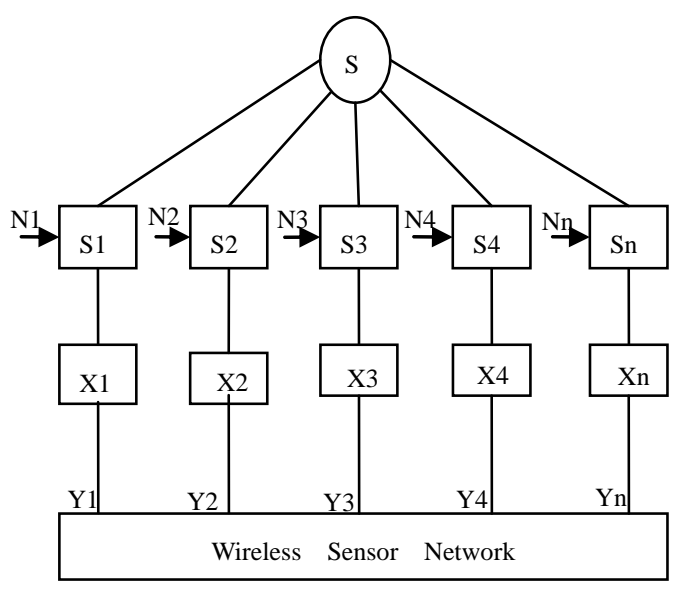

Figure 5. Model for Wireless Sensor Networks - Encoder Part

In Figure 5, The encoder part is labeled as $\mathrm{E}$ and the decoder part is labeled as D in Figure 6. Spatial Correlation based MAC protocol is proposed (CC-MAC) to prevent redundant transmission from closely located neighbors and to regulate the medium access. Spatial Correlation MAC protocol achieves high performance in terms energy.

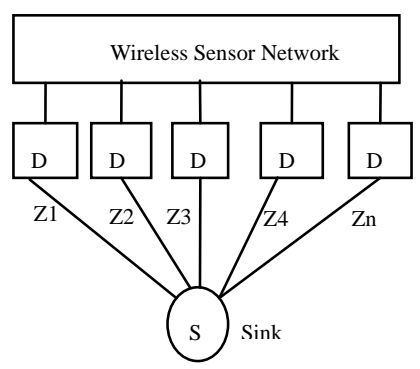

Figure 6. Model for Wireless Sensor Networks - Decoder part 
Total number of nodes available in an event area is N, where the event source is S. Each node in the sensor field observes the noisy version of event information $X_{i}(n), S_{i}(n)$ is spatially correlated to event source $S$. Each node has to encode its observation for the purpose of reporting an event information to sink node. Sink is available at the other end which is responsible for decoding the information to get an estimated value of $\hat{S}$. At a time $\mathrm{n}$, each observed sample is denoted as $\mathrm{X}_{\mathrm{i}}(\mathrm{n})$, equation is formed as,

$$
X_{i}[n]=S_{i}[n]+N_{i}[n]
$$

where the symbol $i$ denotes the spatial location of the node $n_{i}$, i.e. $\left(x_{i}, y_{i}\right), S_{i}(n)$ is the realization of the space-time process $s(t, x, y)$ at time $t=t_{n}$ and $(x, y)=\left(x_{i}, y_{i}\right)$ and $N_{i}(n)$ is the observation noise [1] . $\left\{\mathrm{N}_{\mathrm{i}}(\mathrm{n})\right\}$ is a sequence of i.i.d Gaussian random variables of zero mean and variance $\sigma_{N}^{2}$. We assume that the noise each sensor node encounters is independent of each other, i.e., $\mathrm{N}_{\mathrm{i}}(\mathrm{n})$ and $\mathrm{N}_{\mathrm{j}}(\mathrm{n})$ are independent .Each observation $\mathrm{Xi}(\mathrm{n})$ is then encoded into Yi(n) by the source-coding at the sensor node as,

$$
Y_{i}[n]=f_{i}\left(X_{i}^{[n]}\right)
$$

The information is transferred to the sink through the network. The sink on the other hand decodes the received data to reconstruct an estimation $\hat{S}$ of the source $\mathrm{S}$.

$$
\hat{S}=g\left(Y_{1}\left[n_{1}\right], \ldots, Y_{1}\left[n_{\tau}\right] ; \ldots ; Y_{N}\left[n_{1}\right], \ldots, Y_{N}\left[n_{\tau}\right]\right)
$$

Based on the data received from $\mathrm{N}$ nodes in the event area over a time period $\tau$, the time difference between $t_{n 1}$ and $t_{n \tau}$ is expressed as,

$$
\tau=t_{n_{\tau}}-t_{n_{1}}
$$

\section{Spatial Correlation Based Iterative Node Selection Algorithm (SCINS)}

Node selection technique is used to identify the representative nodes resulting in minimum energy consumption in WSN. For better node selection, Spatial Correlation Based 


\section{Al Macrothink}

Network Protocols and Algorithms

ISSN 1943-3581

2011, Vol. 3, No. 4

Iterative Node Selection (SCINS) algorithm is introduced in this paper. The INS algorithm running at sink, determines the minimum number of representative node to complete the task based on the distortion constraint $\mathrm{D}_{\max }$. Average distance between the sensor node is determined and informed to all the sensor nodes in the event field. Each node in WSN field then performs spatial correlation based collaborative MAC operation. The INS requires the statistical properties of the node distribution as input. They are density of the network, type of the network and type of node distribution. The selection of locations of correlated points based on a distortion constraint has been analyzed by means of vector quantization (VQ) algorithm.

Code book and partition are initially identified by VQ algorithm. Based on the values of code book and partition the distortion value is reduced. The code book represents the locations of the representative node and partition represents the areas of which representative nodes are responsible. Significant improvement is achieved in distortion by selecting the location of representative node using VQ algorithm. The spatial correlation region is defined as the region in which all the sensor nodes send the readings which are similar in nature and therefore it is enough to send a single report to represent the correlation region. Correlation Radius and Correlation Region are two important observations in spatial correlation based iterative node selection algorithm.

$>$ Correlation Radius $\quad-\quad$ It is represented by $r_{c o r r}$

It is the radius of correlation region

$>$ Correlation Neighbor - A node $n_{j}$ is said to be the correlation neighbor of node $n_{i}$ if the distance $d_{i, j}$ of the node $n_{i}$ is smaller than $r_{\text {corr }}$

Pseudo code for the proposed model is

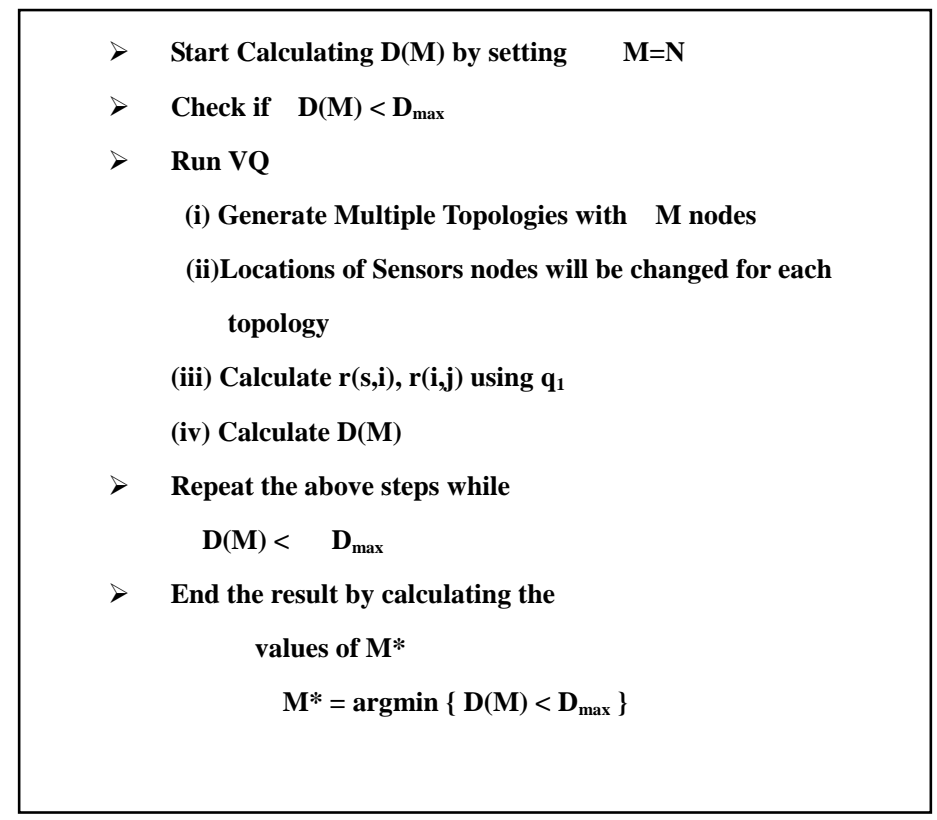




\section{Results and discussion}

A Novel Energy Efficient Routing Protocols for Wireless Sensor Networks using Spatial Correlation based Collaborative Medium Access Control combined with Hybrid MAC has been implemented using Ns2. The parameters such as End to End delay, Packet Delivery Ratio and Throughput, Energy Consumption have been compared with AODV, DSR \&DSDV, AOMDV. Parameters such as Network area, Initial energy, and Transmitting power, Receiving power, Idle power, Data rate and Bandwidth values are tabulated in Table I which have been used in the proposed model.

Table 1. Simulation Parameters

\begin{tabular}{|c|l|l|}
\hline S.No & \multicolumn{1}{|c|}{ Parameters } & \multicolumn{1}{c|}{ Sizes } \\
\hline \hline 1. & Network Area & $1500 \mathrm{X} 1500$ \\
\hline 2. & Number of Nodes & 100 \\
\hline 3. & Packet Length & 250 bytes \\
\hline 4. & Initial Energy & 1000 joules \\
\hline 5. & Bandwidth & $2 \mathrm{MHz}$ \\
\hline 6. & Data Rate & $1 \mathrm{Mbps}$ \\
\hline 7. & Transmitting Power & $1 \mathrm{~mW}$ \\
\hline 8. & Receiving Power & $1 \mathrm{~mW}$ \\
\hline 9. & Idle Power & $1 \mathrm{~mW}$ \\
\hline 10. & Sleep Power & $0.001 \mathrm{~mW}$ \\
\hline
\end{tabular}

\subsection{Comparison of End to End delay with AOMDV,AODV,DSR, DSDV}

End to End delay is defined as the ratio between sum of individual data packet delay to the total number of data packets delivered.

End to End delay $=[($ Sum of Individual data packet delay $) /($ Total number of data Packets delivered)] 


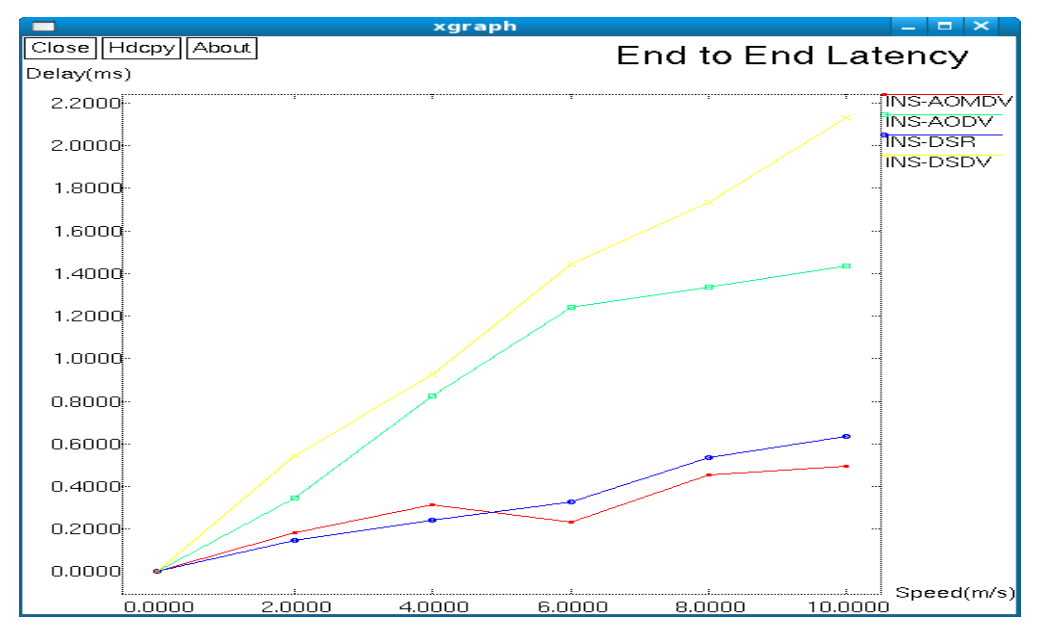

Figure 7. End to End delay Vs Speed

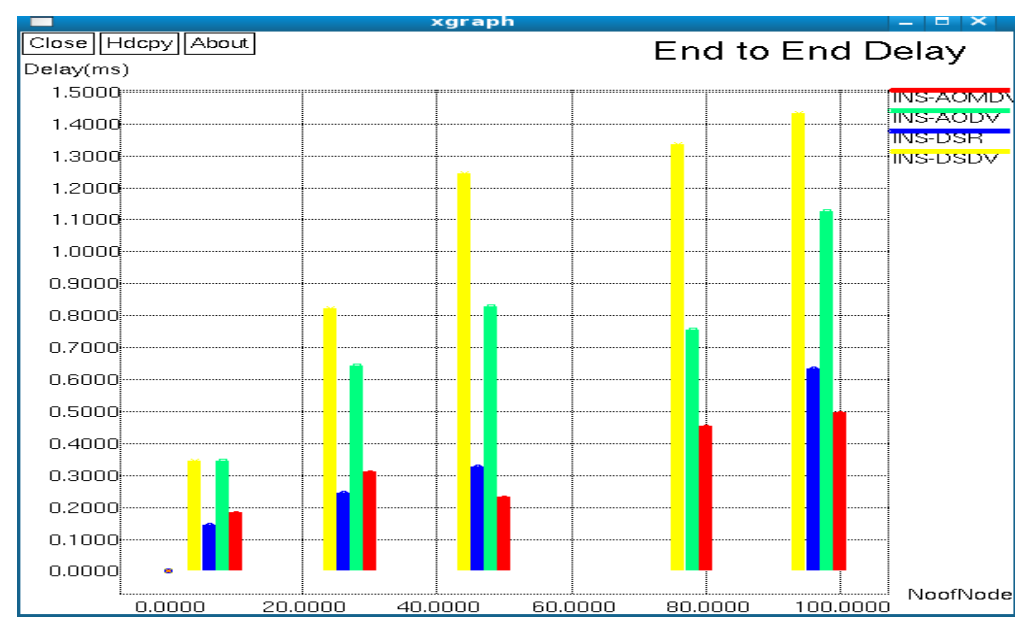

Figure 8. End to End delay Vs No.of Nodes

Fig.7 \& 8 show the variation of End to End delay against speed and No.of Nodes.

It is observed from Figure 7 and 8 that as simulation speed and No.of nodes are increased, the End to End delay with DSR and DSDV and AODV is higher than that of AOMDV. This is because DSDV is applicable for less dense network. DSR is designed for multihop wireless networks but AODV is a routing protocol which has ability to create a route to destination only on demand but AOMDV can find alternate route if the current link has broken.

\subsection{Comparison of Packet Delivery Ratio with AOMDV,AODV,DSR,DSDV}

Packet Delivery ratio is the percentage of the ratio between total number of data packets successfully delivered to the total number of data packets sent.

$\mathrm{Pdr}=[($ Total number of data packets successfully delivered $) /($ Total number of data 
packets sent )]*100\%

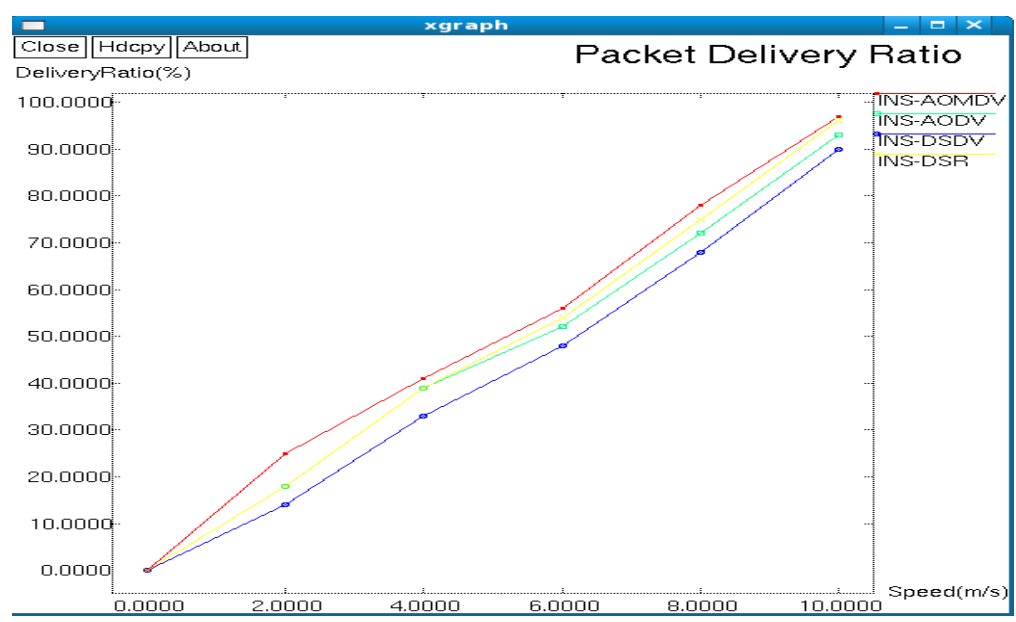

Figure 9. Packet Delivery Ratio Vs Speed

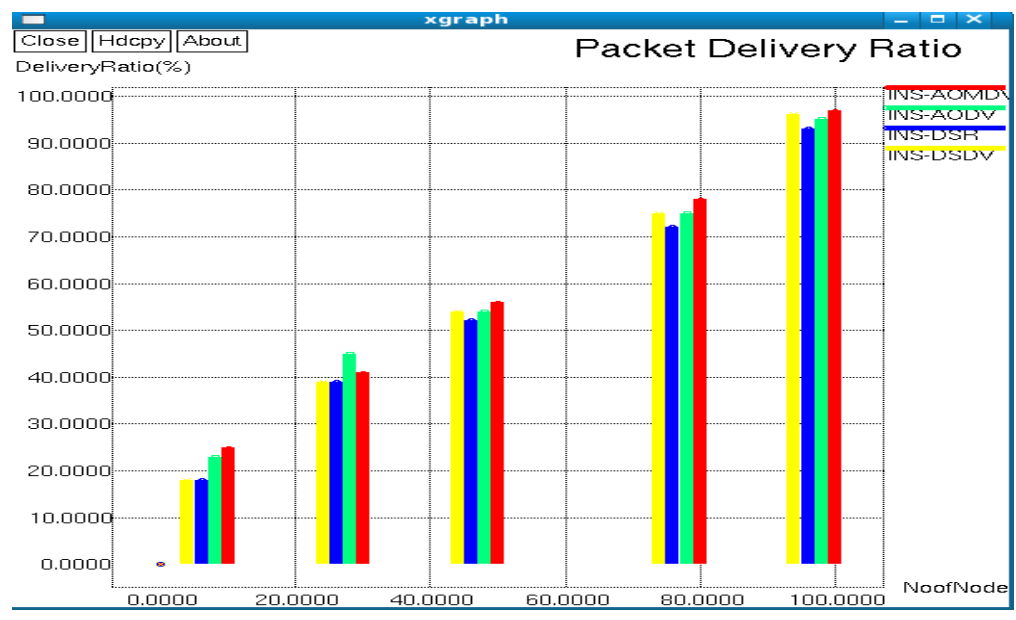

Figure 10. Packet Delivery Ratio Vs No.of Nodes

Figure $9 \& 10$ show the variation of Packet Delivery Ratio against Speed and No. of Nodes

It is observed from Figure 9 and 10 that as simulation speed and No.of nodes are increased, the Packet Delivery Ratio with AODV,DSR and DSDV is lesser than that of AOMDV. This is because DSR uses multiple route cache entries per destination and in DSDV, periodically advertises a node's interconnection topology with the other nodes. AODV uses traditional routing tables, one entry per destination.

\subsection{Comparison of Throughput with AOMDV,AODV,DSR,DSDV}

Throughput is defined as the number of packets delivered at sink node per time unit 


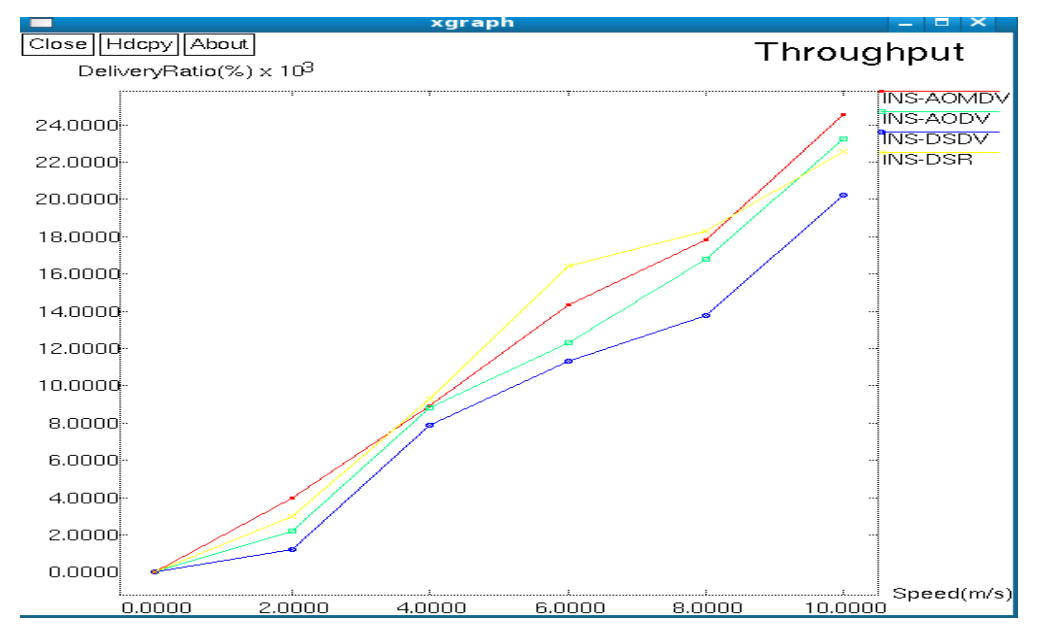

Figure 11. Throughput Vs Speed

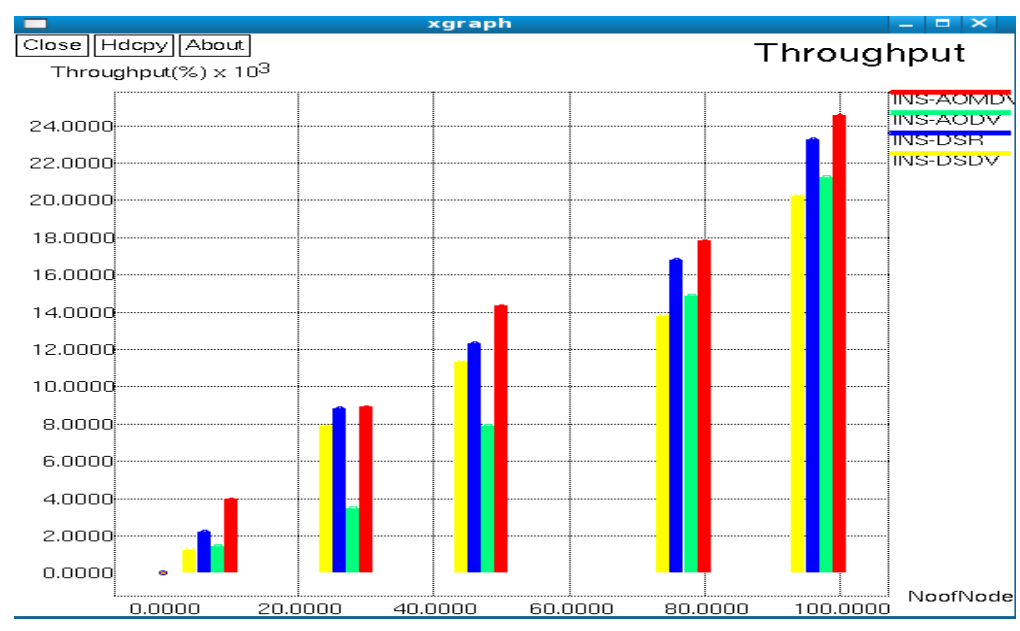

Figure 12. Throughput Vs No.of Nodes

Figure 11 and 12 show the variation of throughput against speed and No.of nodes

It is observed from Figure 11 and 12 that as simulation speed and No.of nodes are increased, throughput with AODV, DSR and DSDV is lesser than that of AOMDV. This is because AODV uses a broadcast route discovery algorithm and then Unicast route reply message. AOMDV outperforms AODV due to its ability to search for alternate route. AOMDV outperforms AODV due to its ability to search for alternate route.

\subsection{Comparison of Energy Consumption with AOMDV, AODV, DSR, DSDV}

Energy Consumption is defined as the ratio between the sum of energy expended by each node to the total number of data packets delivered.

Energy Consumption $=[($ Sum of Energy expended by each node $) /($ Total number of 


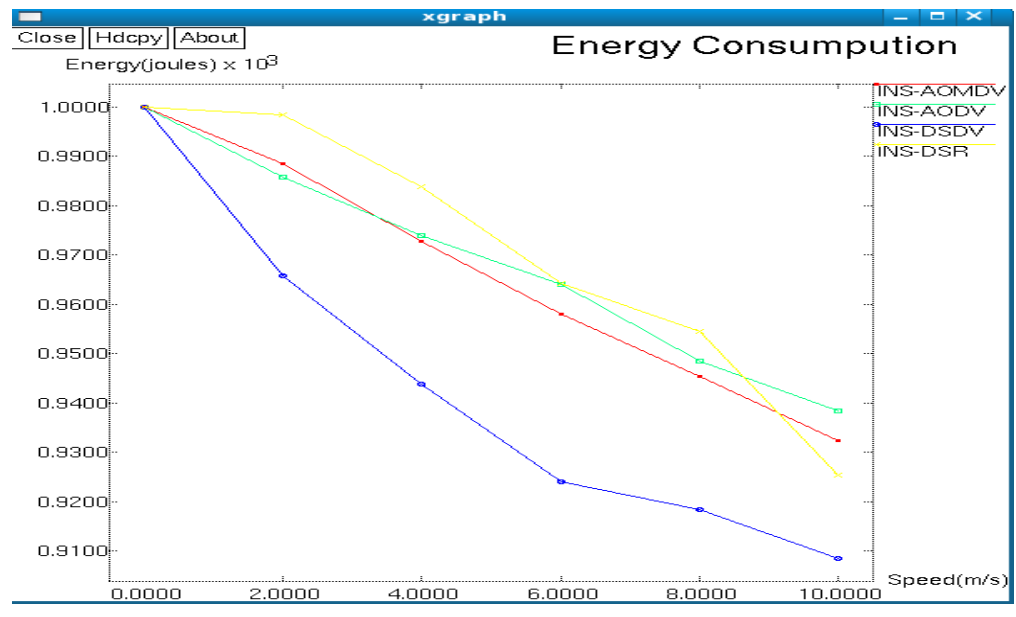

Figure 13. Energy Consumption Vs Speed

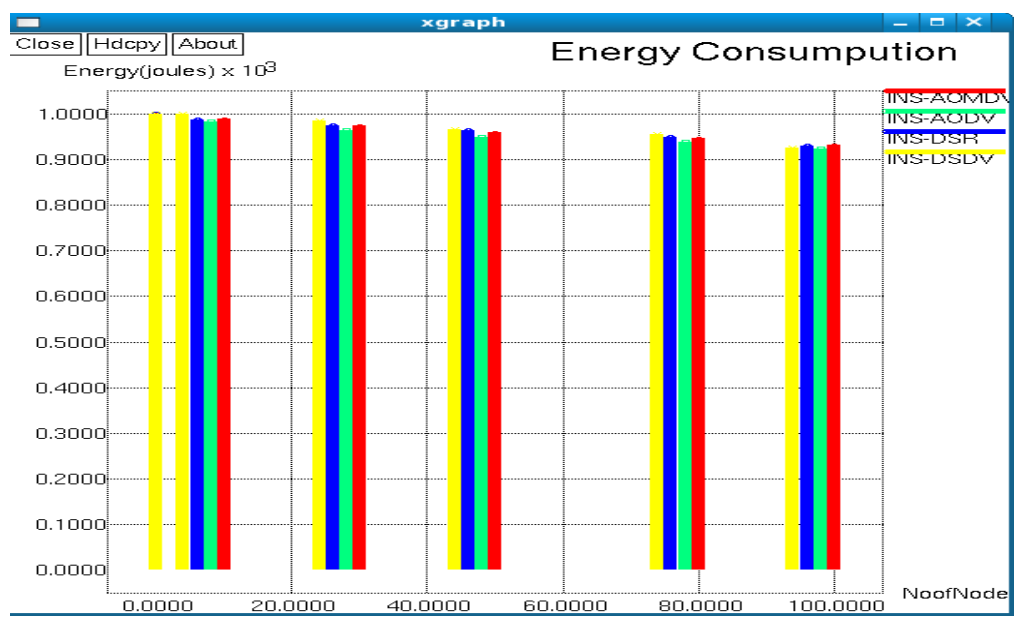

Figure 14. Energy Consumption Vs No.of Nodes

Figure 13 and 14. show the variation of Energy Consumption against Speed and No. of Nodes

It is observed from Figure 13 and 14 that as simulation speed and No. of nodes are increased, Energy Consumption with AODV,DSR and DSDV is higher than that of AOMDV. AOMDV shows the lesser energy consumption. This is because DSR needs support from MAC layer to identify the link failure. In DSDV, an additional table is maintained by the mobile nodes that stores the data sent in the incremental routing information packets but in AODV, a route between the source and destination node is found only when they are needed but AOMDV allows intermediate nodes to reply for RREQ, which still selecting disjoint paths.

From Figure 7,8,9,10,11,12,13,14, it is observed that the proposed a novel energy efficient routing protocols for wireless sensor network using spatial correlation based collaborative medium access control Combined with Hybrid MAC shows AOMDV performs better with 
higher Packet Delivery Ratio, lesser End to End delay and better Throughput, lesser Energy Consumption than that of DSR and DSDV algorithms and AODV algorithms.

\section{Conclusion and Future Work}

A Novel Energy Efficient Routing protocols for Wireless Sensor Network using Spatial Correlation based Collaborative Medium Access Control combined with Hybrid MAC is proposed in this paper. The spatial correlation between the sensor nodes is considered in the proposed algorithm. Using Ns2, Wireless Sensor Networks are simulated and the proposed algorithm is implemented with spatial correlation based AOMDV, AODV, DSDV, DSR. The spatial resolution of nodes are controlled by deactivating the redundant nodes. The performance of $\mathrm{CC}$ - MAC combined with Hybrid MAC is analyzed using Vector Quantization method. It yields better performance to achieve Energy Efficiency. Parameters such as, End to End Delay, Packet delivery Ratio, Throughput and Energy Consumption are taken into account. Among the routing protocols AOMDV gives better results than DSR, DSDV, AODV. By reducing the redundant data from redundant nodes the spatial correlation based method proves that it is the suitable technique to attain energy efficient in WSN . In future work, field of grid and cluster head based algorithm will be detailed. Also, more number of routing algorithms will be considered to accomplish the energy efficient operation in a better way. In future, various network topology may be considered to proceed the work in different directions.

\section{References}

[1] Mehmet, C.,Vuran, Ian F., Akyildiz, "Spatial Correlation - Based Collaborative Medium Access Control in Wireless Sensor Networks", IEEE / ACM Transaction on Networking, 2006, vol. 14, no. 2,http://dx.doi.org/10.1109/TENT.2006.872544.

[2] Guoqiang Zheng, Shengyu Tang, "Spatial Correlation Based MAC Protocol for Event Driven Wireless Sensor Networks", Journal of Networks, 2011,vol. 6, no. 1, http://dx.doi.org/ 10.4304/jnw.6.1.121-128.

[3] Zheng Teng, Ki-Il Kim, "A survey on Real-Time MAC protocols in Wireless Sensor Networks, Journal of communication and networks", 2010, vol.2, pp.104--112, http://dx.doi.org/10.4236/cn.2010.22017.

[4] Ilker Demirkol, Cem Ersoy, Fatih Alagoz, "MAC Protocols for Wireless Sensor Networks:Asurvey",IEEECommunicationMagazine,2006,http://dx.doi.org/10.1109/MCOM. 2006.1632658.

[5] Khaled A., Arisha, Moustafa A., Youseef, Mohamed F., Younis, "Energy Aware TDMA-Based MAC for Sensor Networks", In Proceedings of the IEEE Integrated Management of Power Aware Communications, Computing and Networking (IMPACCT), 2002, http://dx.doi.org/10.1.1.120.9653. 


\section{Macrothink}

[6] M., C., Vuran, O., B., Akan, and I., F., Akyildiz " Spatio-Temporal Correlation: Theory and Applications for Wireless Sensor Networks", Comput. Networks Journal. (Elsevier), 2004, vol. 45, no. 3, pp. 245--259, http://dx.doi.org/10.1016/j.comnet.2004.03.007.

[7] Samir R., Das, Charles E., Perkins, Elizabeth M., Royer, "Performance Comparison of Two On-demand Routing Protocols for Adhoc Networks", Proceedings of the IEEE Conference on Computer Communications (INFOCOM), Tel Aviv, Israel,2000.

[8] Ghalib A., Shah, Muslim Bozyigit, "Exploiting Energy Aware Spatial Correlation in Wireless Sensor Networks", $2^{\text {nd }}$ International Workshop on software for Sensor Networks (Sensor Wave 2007), 2007.

[9] Y., Linde, A., Buzo, and R., M., Gray , “ An Algorithm for Vector Quantizer design”, IEEE Trans.Commun.,vol.COM-28,1980,no.1,pp.84-95, http://dx.doi.org/ 10.1109/TCOM.1980. 1094577.

[10] Radhika D., Joshi, Priti P., Rege, "Energy Aware Routing in Ad Hoc Networks", 6 WSEAS International Conference on CIRCUITS, SYSTEMS, ELECTRONICS, CONTROL \& SIGNAL PROCESSING”, CAIRO, Egypt, 2007.

[11] The Network Simulator-ns-2. [Online]. Available: http://www.isi.edu/nsnam/ns/index.html

[12] Heping Wang, Xiaobo Zhang, Farid Nait-Abdesesselam, "Cross Layer Optimized MAC to support Multihop QOS routing for Wireless Sensor Network", IEEE Transactions on Vehicular Technology, 2010,vol. 59,no.5, http://dx.doi.org/10.1109/TVT.2010.2042185

[13] Biradar S.R.,Koushik Majumder,Subir Kumar Sarkar, Puttamadappa C., "Performance Evaluation and Comparison of AODV and AOMDV ". International Journal on Computer Science and Engineering, 2010, vol. 02, no. 02, pp-373-377.

\section{Copyright Disclaimer}

Copyright reserved by the author(s).

This article is an open-access article distributed under the terms and conditions of the Creative Commons Attribution license (http://creativecommons.org/licenses/by/3.0/). 